To the Editors:

\title{
A case of sarcoidosis
}

In May 2000, a 30-year old nurse presented at the Central Chest Clinic, Colombo, with progressive shortness of breath and a non-productive cough of 6 months' duration. She had been extensively investigated in 1999 for pyrexia of unknown origin at the National Hospital of Sri Lanka. Clinical examination at the time showed hepatosplenomegaly. The chest radiograph did not show any abnormality at this stage. Blood cultures and thick films for malarial parasites were repeatedly negative, as was the tuberculin test. The fever spontaneously subsided and she was discharged.

In January 2000 , the patient again presented at the National Hospital with an unproductive cough and breathlessness. The chest radiograph at this stage revealed fine nodular opacities mainly concentrated in both upper and mid zones. A repeat tuberculin test remained negative and pulmonary function tests showed a mild restrictive defect with markedly reduced diffusing capacity (DLCO $34 \%$ ). Fibreoptic bronchoscopy did not show any intrabronchial pathology and washings were negative for malignant cells and acid-fast bacilli. Transbronchial lung biopsy was unsuccessful.

A the Central Chest Clinic, Colombo in May 2000 careful palpation of the neck revealed a small rubbery lymph node just lateral to the cricothyroid cartilage. Systemic examination did not show any other abnormality except for hepatosplenomegaly. The chest radiograph showed widespread fine nodular opacities of much greater profusion compared to the radiograph of January 2000.

Fine needle aspiratoin biopsy of the enlarged lymph node was performed and it revealed granulomatous lymphadenitis with no caseation. Taking the clinical, radiological and laboratory features into account sarcoidosis was considered highly likely (1).

An open lung biopsy was performed in June 2000. Histology again showed granulomatous lesions with no caseation. Special stains done on the lung tissue were nagative for mycobacterial, fungal and parasitic organisms. A part of the lung biopsy specimen was despatched for mycobacterial culture. The culture was negative.
Serum calcium, phosphate and immunoglobulins were within the normal range. The CD4 count was normal. Slit lamp examination of the eyes did not show any evidence of uveitis. An electrocardiogram was normal. The patient was commenced on prednisolone $30 \mathrm{mg}$ daily in July 2000 . No other drugs were given as this could have confused the diagnosis.

After 6 weeks of prednisolone, there was a significant reduction in the severity of breathlessness and a repeat chest radiograph in September 2000 showed significant improvement. The diffusing capacity (DLCO) rose from $34 \%$ to $48 \%$ at end October. The patient remains well with only minimal symptoms on a maintenance dose of prednisolone.

Sarcoidosis should be considered in any young patient presenting with a compatible clinical and radiological picture (1). It affects vital organs and leads to irreversible functional impairment unless treated early with prednisolone. Sarcoidosis mimics tuberculosis closely, and the consequences of a wrong diagnosis would be disastrous for the patient. Empirical administration of antituberculosis drugs to undiagnosed or improperly diagnosed lung disease is widely practiced in Sri Lanka and this should be avoided. Histological diagnosis of obscure lung pathology should be a routine matter.

Sarcoidosis is considered to be rare in Sri Lanka, but this may simply be due to a low index of chinical suspicion (2), to the difficulties in obtaining transbronchial or open lung biopsies in our country, and the uncritical use of antituberculosis drugs. This patient happens to. be the first case of sarcoidosis reported in Sri Lanka, though a few Sri Lankans living in Sri Lanka have been diagnosed as sarcoidosis when they sought treatment abroad.

\section{References}

1. James DG, Turiafj HY. Description of sarcoidosis: Report of sub-committee on classification and definition Annals of the New York Academy of Sciences 1976; 278: 742.

2. Scadding JG, Mitchell DN, Sarcoidosis, London: Chapman and Hall, 1985.

P N B Wijekoon, Chest Physician, Central Chest Clinic, Colombo; M P Kumarasinghe, Associate Professor in Pathology, Faculty of Medicine, University of Colombo; and M D P Hidelaratchi, Medical Officer, Central Chest Clinic, Colombo. (Corresponding author P N BW, telephone +94 1 699550, email: pnbw@slt.lk) 